\title{
Design Optimization of B-series Marine Propeller using NSGA-II, Iterative and Gekko Algorithm
}

\author{
S. M. Munawar Mahtab, Debasish Roy, M. S. Rabbi*, Md. Iftekharul Alam
}

Department of Mechanical Engineering, Chittagong University of Engineering and Technology, Chattogram-4349, Bangladesh.

Received: July 02, 2021, Revised: August 17, 2021, Accepted: August 23, 2021, Available Online: August 31, 2021

\begin{abstract}
The design of a propeller plays a significant role in naval architecture. Optimization of various design factors is the primary concern for effective and efficient propulsion. This study investigates the optimization of the B-series marine propellers using three different methods, i.e. (i) a non-linear constrained single-objective optimization approach using the Non-Dominated Sorting Genetic Algorithm (NSGA-II), (ii) a python package for dynamic optimization based optimization software 'Gekko', (iii) an iterative approach and results were compared with each other. Efficiency is considered as the single objective function whereas three constraints are imposed: cavitation, thrust and strength. Analogous characteristics have been found in the comparison of results from all three methods. Comparing the various factors, this study suggests that, Gekko can be used as the optimization algorithm.
\end{abstract}

Keywords: Optimization, B-series Propeller, NSGA-II, Iterative, Gekko.

This work is licensed under a Creative Commons Attribution-Non Commercial 4.0 International License.

\section{Introduction}

In naval architecture, the propeller design plays a significant role in naval architecture. Marine propellers are operated in nonuniform flow of water and thus complex to be modelled the environment and analysed. Performance of a marine propeller depends on numerous parameters such as thrust, cavitation, material strength, geometry, shape etc. Optimization of the theoretically designed marine propellers is a way to increase the performance and hence, the efficiency. The constrained single objective optimization has been used in this study for optimization. In this method, a single parameter considered as an objective and other parameters can be taken as design constraints [1].

Among numerous algorithms, Genetic algorithm i.e. NonDominated Sorting Genetic Algorithm (NSGA-II), and iterative algorithm follow the evolutionary approach to investigate the optimized solution for a given problem taking in consideration various constraints and the main objective. Xie [2] adopted multi-objective optimization approach where efficiency ratio and thrust coefficient were taken as objectives. To avoid the dangerous effects of increased cavitation, it was kept as a constraint. Gaafary et al. [3] developed an algorithm to find out optimum design parameters of B-series propellers where the single objective optimization approach has been adopted keeping propeller thrust, material strength, and cavitation as constraints to maximize efficiency. Theoretical findings were compared with the results found from the commercial software.S. Mirjalili et al. [4] used multi-objective optimization where efficiency was taken as a maximization function and cavitation was taken as a minimization function. It was found that the efficiency increases proportional to the number of blades up to a limit of 5 blades and cavitation also decreases proportional to the number of blades. Windyandari et al. [5] investigated the Indonesian purse seine boats to find optimized design of marine propellers which will improve thrust performance of the traditional purse seine boats.
Oosterveld et al. [6] performed a multi-regressional analysis using some open-water test data of B-series propellers. These regression analysis data can be used to design the propeller in marine engineering. Suen et al. [7] used genetic algorithm to find out optimum design parameters for B-series marine propellers. Main objective of the optimization was maximization of efficiency using non-linear constraints. The results achieved using genetic algorithm was compared with results of iteration method. It was observed that genetic algorithm yielded higher efficiency and more reliable result than the iteration method. Benini et al. [8] carried out a multi-objective design optimization experiment for marine propellers. The two objectives of the optimization were maximization of open-water efficiency and thrust coefficient. Result of the study showed that the two objectives conflicts each other.

In the present study, optimization of the B-series marine propeller is investigated using dynamic optimization package Gekko, based on python programming language. To verify the technique, the popular optimization methods stated in the review, NSGA-II and iterative algorithms have also been conducted and consequently. In this optimization procedure, a single objective optimization approach was adopted where cavitation, material strength and thrust requirements were defined as constrains. The objective function was maximization of efficiency. To make a comparison with the three methods the issue was imposed as single objective since the iterative algorithm was specially designed for constrained single objective optimization. The results were compared considering computing cost, time, and efficiency.

The mathematical representation of design objective and constraints are mentioned in Section 2. Various optimization algorithms are explained in this section as well. Section 3 contained the numerical simulation parameters. Depiction of result and their illustrations are presented in Section 4. Conclusions are drawn in Section 5. 


\section{Optimization Problem}

\subsection{Design Objective}

To optimize the marine propeller, maximization of efficiency is the main objective function, considered in this study. The open water efficiency of marine propellers is calculated using the following equations:

$\eta=\frac{J}{2 \pi} \cdot \frac{K_{T}}{K_{Q}}$

and

$J=\frac{V_{S}(1-w)}{n D}$

where $K_{T}$ and $K_{Q}$ are thrust coefficient and torque coefficient respectively. $J$ is the advanced coefficient and $V_{S}$ is the service speed.

\subsection{Design Constraints}

In the constrained single objective optimization technique, important parameters that are interrelated with the performance of a marine propeller needed to be defined as constraints. Cavitation is one of the crucial parameters depends on blade area ratio. Cavitation can be avoided by increasing the blade area ratio and the minimum blade area ratio can be calculated using the following equation [9]

$\left[\frac{A_{E}}{A_{O}}\right]_{\min }=\frac{(1.3+0.3 Z) T}{\left(P_{O}-P_{V}\right) D^{2}}+K$

where, $K=0.1,0.2$ for twin-screw and single-screw ships, correspondingly.

$Z=$ number of blades

$P_{O}=$ the centreline of the propeller shaft static pressure

$P_{V}=$ vapour pressure

$D=$ propeller diameter

So, the cavitation constraint for the optimization is

$\left[\frac{A_{E}}{A_{O}}\right] \geq\left[\frac{A_{E}}{A_{O}}\right]_{\text {min }}$

A minimum amount of generated propeller thrust is also required to achieve a better overall performance. To ensure the thrust requirement, a thrust constraint is needed for the optimization. The generated propeller thrust $\left(T_{C A L}\right)$ must be larger than the required thrust $\left(T_{R}\right)$ and can be formulated as [3]:

$T_{C A L}=K_{T} \rho n^{2} D^{4}$

$T_{R}=\frac{R_{T}}{N_{p}\left(1-t_{d}\right)}$

where, $\rho=$ seawater density

$R_{T}=$ total resistance of the ship

$N_{p}=$ number of propeller

$t_{d}=$ thrust deduction

To maintain optimum strength of the propeller blades, the minimum blade thickness ratio should be considered in the optimization process. The minimum blade thickness to diameter ratio can be determined using the following formula [10]:

$$
\left[\frac{t_{\min }}{D}\right]_{0.7 R}
$$$$
=0.028+0.21 \sqrt[3]{\frac{[3183.87-1508.15(P / D)] P_{s}}{1266652.04 n D^{3}\left(S_{c}+20.9 D^{2} n^{2}\right)}}
$$

where, $\left[\frac{\mathrm{t}_{\min }}{\mathrm{D}}\right]_{0.7 \mathrm{R}}=$ minimum blade thickness ratio.

Using this equation and considering geometry of the propellers the blade thickness should satisfy the following criterion:

$\left[\frac{t}{D}\right]_{0.7 R} \geq\left[\frac{t_{\min }}{D}\right]_{0.7 R}$

This is the strength constraint for the optimization process.

\subsection{Optimization methods}

\subsubsection{NSGA II}

Genetic algorithms follow an evolutionary approach to find out optimized solution for a problem [11]. In this single objective optimization problem, a special type of genetic algorithm is applied. Non-Dominated Sorting Genetic Algorithm uses a nondominance arranging for the parent populations. Using the random parent population, new generations are found and sorted according to their fitness. Eventually the best possible values of the objective function are given as output. The algorithm uses a principle, i.e. the elitist has the right to carry out the procreation process for the next generation. Where an explicit diversity preservation mechanism is used that emphasizes the nondominated solutions among all other solutions.

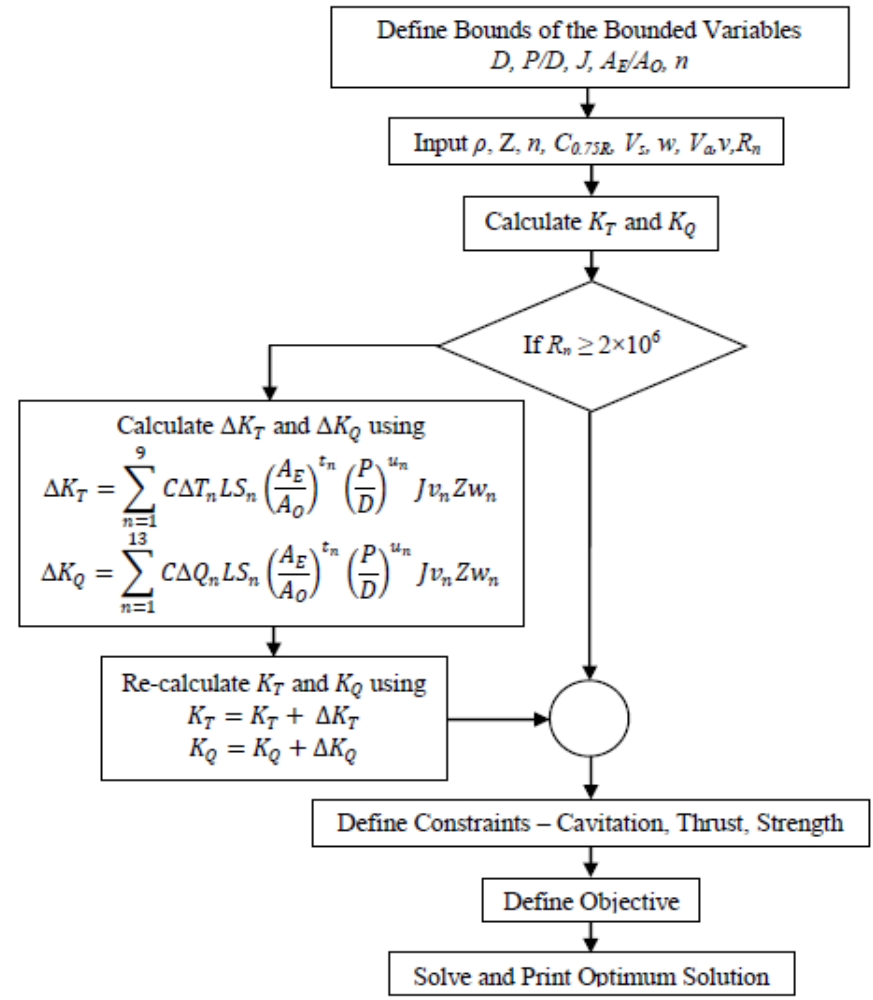

Fig. 1 Flowchart of the methodology followed in this study 


\subsubsection{Iterative Algorithm}

Gaafary et al. [5] developed a special program that follows an iterative approach and specially designed for constrained single objective optimization to find optimum solution for Bseries marine propellers.

\subsubsection{Gekko}

Gekko is an open-source python package that facilitates the use of python programming language in the field of dynamic optimization [11]. It is an Algebraic Modelling Language that facilitates the use of nonlinear model predicative control (NMPC), real-time optimization (RTO), moving horizon estimation (MHE), and dynamic simulation. Since propeller optimization problem is constrained non-linear problem so, the present study imposes this software package to address this issue. Method used in this study is illustrated by flowchart in Fig. 1.

Using the constraints and algorithms, numerical simulation has been done.

\section{Numerical Simulation}

Determination of the design parameters is the key factor for any optimization technique. Following the literature [5] the input data are listed in Table 1, the range of the bounded variables is given in Table 2, and to conduct the experiment for NSGA II technique, the input values are listed in Table 3.

Table 1 Input variable for numerical computation

\begin{tabular}{|l|c|}
\hline \multicolumn{1}{|c|}{ Input Parameter } & Value \\
\hline Total resistance, $R_{T}$ & $35000 \mathrm{kN}$ \\
\hline Service speed, $V_{S}$ & $12 \mathrm{~ms}^{-1}$ \\
\hline Number of propellers, $N_{P}$ & 2 (double screw) \\
\hline Number of blades, $Z$ & 4 \\
\hline Propeller immersion, $P_{C L}$ & $2.5 \mathrm{~m}$ \\
\hline Wake, $W$ & 0.2 \\
\hline Thrust deduction, $t_{d}$ & 0.15 \\
\hline Permissible stress, $S_{C}$ & $265 \mathrm{MPa}$ \\
\hline Height of propeller aperture, $H_{p a p}$ & 1.2 \\
\hline
\end{tabular}

Table 2 Range of bounded variables

\begin{tabular}{|l|c|}
\hline \multicolumn{1}{|c|}{ Parameter } & Range \\
\hline Diameter, $D$ & $0.2 \sim 1.0$ \\
\hline Expanded area ratio, $A_{E} / A_{O}$ & $0.3 \sim 0.8$ \\
\hline Pitch, P/D & $0.25 \sim 1.4$ \\
\hline Advanced co-efficient, $J$ & $0.2 \sim 1.2$ \\
\hline Rotating speed, $n$ & $5 \sim 15$ \\
\hline
\end{tabular}

Table 3 Parameters for NSGA II computation

\begin{tabular}{|l|c|}
\hline \multicolumn{1}{|c|}{ Parameter } & Value \\
\hline Max generations & 100 \\
\hline Population & 100 \\
\hline Mutation probability & 0.05 \\
\hline Distribution index & 20 \\
\hline Crossover index & 20 \\
\hline
\end{tabular}

\section{Results and Discussion}

The calculations were done for 4-bladed B-series marine propeller. For each algorithm, 11 solutions were available. The advanced co-efficient, $J$ plays a significant role in the performance of propeller. Decision variables are plotted in Fig. 2. It is found that, the $K_{T}$ and $K_{Q}$ shows analogous behaviour for all techniques. As the value of $J$ increases, the value of $K_{T}$ and $K_{Q}$ decreases. $K_{T}$ shows higher value for Gekko algorithm than the others.

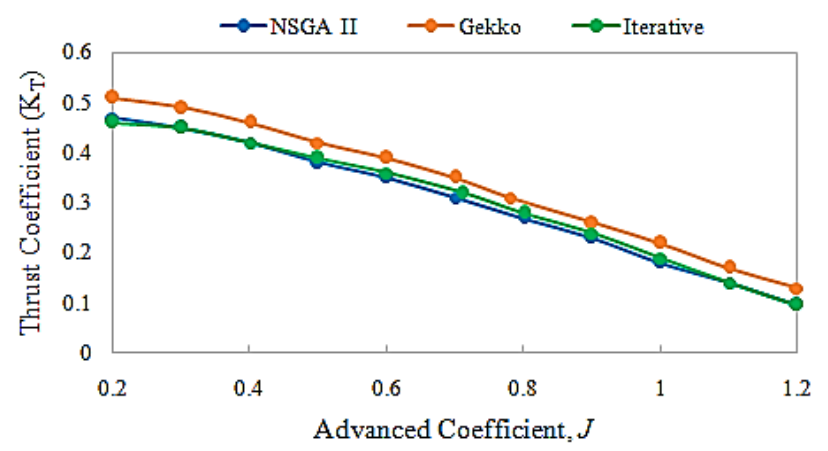

(a)

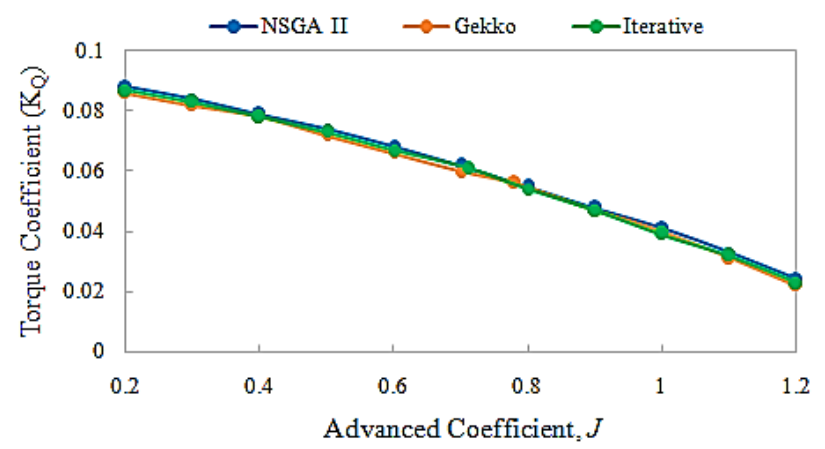

(b)

Fig. 2 (a) Thrust and (b) Torque co-efficient variation for different algorithms

It has been found that the open water diagram provides the insight of the performance of any marine propeller optimization technique. Fig. 3 illustrates the open water diagram for all algorithms used in this study. It can be seen that, with the increasing value of co-efficient $J$ upto 0.8 , the efficiency increases and for the higher value of $J$, it decreases gradually. Among 11 solutions, the best solutions are listed in Table 4 and the significant parameters are plotted in Fig. 4.

A single objective optimization approach is considered in this study and efficiency maximum is the only concern in this optimization technique. Fig. 5 depicts the trend of the efficiency for all algorithms.

Table 4 and Fig. 5 indicated that the efficiency reaches at its maximum value for the value of $J, 0.8$. Maximum efficiency is found for the iterative method though for the value of 0.8 , it reaches at lowers than others two. From the findings from optimization techniques, it can be said that, for the considered environment and parameters, the variables determining the advanced co-efficient, should be set at the value for maximization of the output. Moreover, Gekko can be used for any optimization technique alongside the popular techniques considering lesser calculation time consumption and easy to handle constrained optimization problems point of view. 
Table 4 Best solutions for each algorithm

\begin{tabular}{|c|c|c|c|c|c|c|c|c|c|c|}
\hline Algorithm & $D$ & $P / D$ & $J$ & $A_{E} / A_{O}$ & $n$ & $\eta$ & $T$ & $Q \quad K_{T}$ & $K_{Q}$ \\
\hline NSGA II & 0.90 & 1.30 & 0.80 & 0.5 & 14.1 & 0.625 & 36303.2 & 6647.8 & 0.27 & 0.055 \\
\hline Iterative & 0.94 & 1.32 & 0.80 & 0.56 & 14.1 & 0.630 & 44963.0 & 8726.1 & 0.28 & 0.054 \\
\hline Gekko & 0.92 & 1.38 & 0.78 & 0.56 & 14.0 & 0.625 & 45612.3 & 8887.8 & 0.27 & 0.055 \\
\hline
\end{tabular}

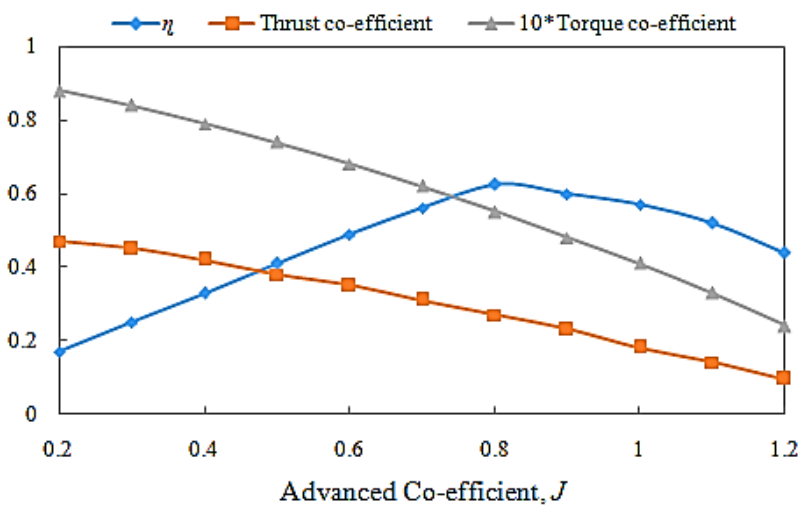

(a)

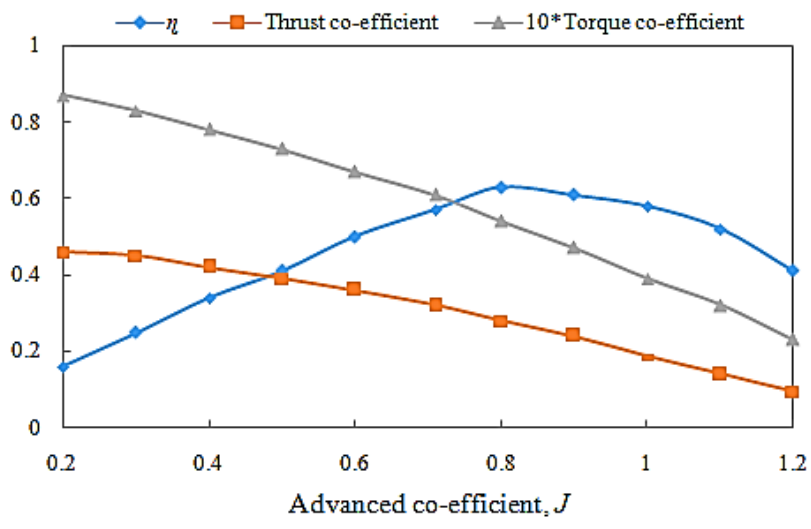

(b)

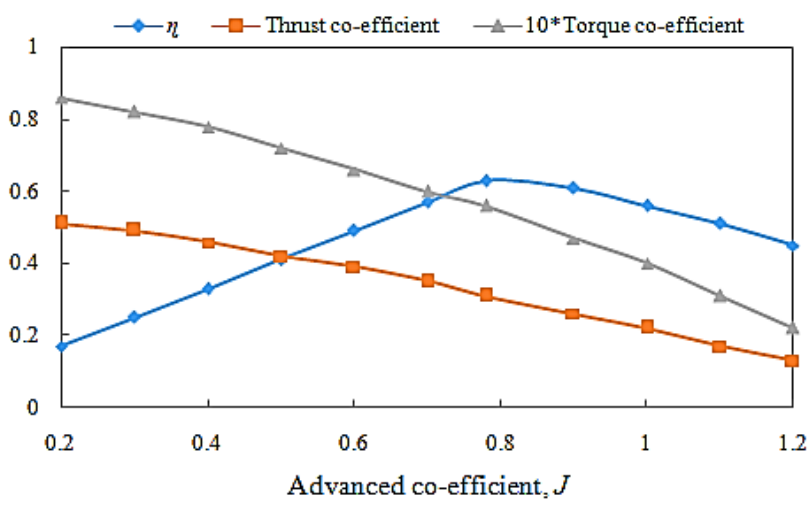

(c)

Fig. 3 Open water diagram for (a) NSGA II, (b) Iterative, (c) Gekko algorithm

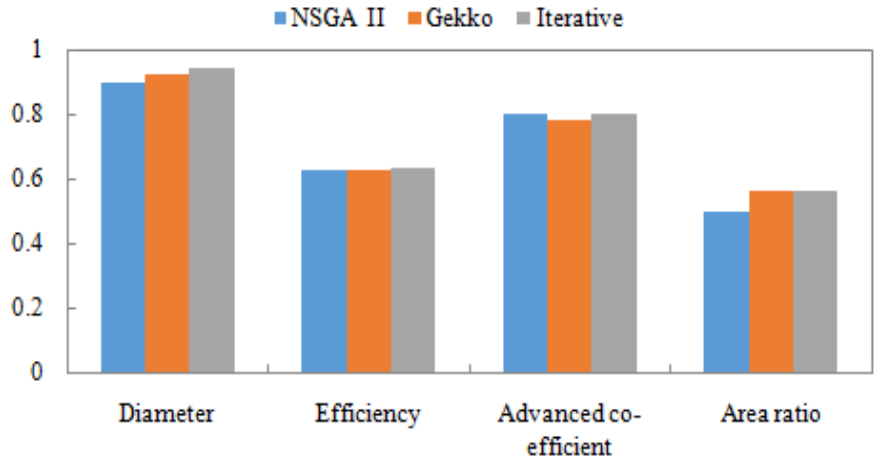

Parameters

Fig. 4 Comparison of bounded variables used in this study for different algorithms

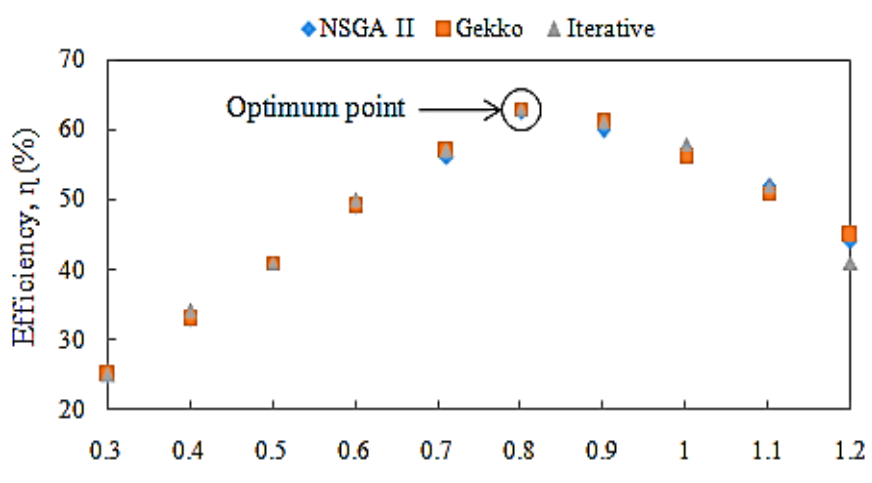

Advanced co-efficient, $J$

Fig. 5 Comparison of efficiency, found for different algorithms

\section{Conclusion}

A comparison of Non-Dominated Sorting Genetic Algorithm (NSGA II), Gekko optimization software package and an iterative algorithm in designing marine propeller is presented in this study. Three different methods yield in values in a close proximity to each other. The results also manifest a great agreement with theoretical characteristics of B-series marine propellers.Results from the three different methods have been compared to get a better method for design optimization of Bseries propellers using single objective optimization. Though the iterative algorithm resulted in better efficiency, Gekko is recommended in terms of less time consumption and easy to handle constrained equations. So, its practice should be increased in the optimization field. 


\section{References}

[1] Savic, D., 2002. Single-objective vs. multiobjective optimisation for integrated decision support. Proceedings of the First Biennial Meeting of the International Environmental Modelling and Software Society 1, 7-12.

[2] Xie, G., 2011. Optimal preliminary propeller design based on multi-objective optimization approach. Procedia Engineering, 16, pp.278-283.

[3] Gaafary, M.M., El-Kilani, H.S. and Moustafa, M.M., 2011. Optimum design of B-series marine propellers. Alexandria Engineering Journal,50(1), pp.13-18.

[4] Mirjalili, S., Lewis, A. and Mirjalili, S.A.M., 2015. Multiobjective optimisation of marine propellers. Procedia Computer Science, 51, pp.2247-2256.

[5] Windyandari, A., Haryadi, G.D. and Zakki, A.F., 2018. Optimization Procedure To Determine The Optimum Propeller Of Traditional Purse Seine Boat. Technology, 9(13), pp.1519-1526.

[6] Oosterveld, M.W.C. and van Oossanen, P., 1975. Further computer-analyzed data of the Wageningen B-screw series. International shipbuilding progress,22(251), pp.251-262.
[7] Suen, J.B. and Kouh, J.S., 1999. Genetic algorithms for optimal series propeller design. WIT Transactions on The Built Environment, 45.

[8] Benini, E., 2003. Multiobjective design optimization of B-screw series propellers using evolutionary algorithms. Marine technology and SNAME news, 40(04), pp.229-238.

[9] Chen, J.H. and Shih, Y.S., 2007. Basic design of a series propeller with vibration consideration by genetic algorithm. Journal of marine science and technology, 12(3), pp.119-129.

[10] Karim, M.M., 2000. A genetic algorithm (GA)-based optimization technique for the design of marine propellers. In Proceedings of the propeller/shafting 2000 symposium. Virginia Beach, USA.

[11] Beal, L.D., Hill, D.C., Martin, R.A. and Hedengren, J.D., 2018. Gekko optimization suite. Processes, 6(8), p.106.

[12] Martínez-Cagigal V., 2021. Non Sorting Genetic Algorithm II (NSGA-II), (https://www.mathworks.com/matlabcentral/fileexchang e/65494-non -sorting-genetic-algorithm-ii-nsga-ii), MATLAB Central File Exchange. Retrieved January 7, 2021. 